\title{
Acciones para elevar el conocimiento de la educación ambiental en la comunidad Natividad del Municipio La Sierpe
}

\author{
Maité Felicia Valdivia Valdés ${ }^{1}$ \\ Julio César Calderón Leiva²
}

\section{Resumen}

F ste trabajo ha constituido una revisión crítica sobre temas relacionados con el medio ambiente y el Edeterioro del mismo, tanto en el mundo como en Cuba y la necesidad de elevar este conocimiento en los habitantes de la comunidad Natividad del municipio de la Sierpe en la provincia Sancti-Spíritus, Cuba. Se realizó un diagnóstico de las principales problemáticas existentes en la comunidad y así seguir modelos que conduzcan al desarrollo sostenible. El trabajo ha propuesto acciones directas que desarrollen los individuos en su medio para mejorar su calidad de vida.

Palabras clave: medio ambiente; deterioro; conocimiento; comunidad; acciones; individuos; calidad de vida.

\section{Summary}

This work is made based on a critical review on issues related to the environment and its deterioration, in the world as well as in Cuba, and the need to raise this knowledge in the inhabitants of the Natividad community of the municipality of La Sierpe in the province Sancti-Spiritus, Cuba. We carried out a diagnostic of the main problems in the community, in order to follow models that lead to sustainable development. The work has proposed direct actions for individuals to develop in their own environment to improve their quality of life.

Keywords: Environment; deterioration; knowledge; community; actions; individuals; life quality.

\section{Introducción}

$\mathrm{C}^{1}$ medio ambiente es objeto de constantes transformaciones que provocan un profundo deterioro del Emismo. Esto ha puesto en peligro la calidad de la vida humana y avanza progresivamente hasta el punto de verse comprometida la supervivencia de la especie humana en el planeta. La utilización de los recursos naturales por el hombre para su subsistencia, se ha convertido en explotación irracional. Ello ha alterado la armónica dinámica de los ecosistemas, que trae aparejado la desaparición de sus valores, al no ser compatible el ritmo de explotación con el de recuperación natural.

Uno de los componentes mayormente afectados es la diversidad biológica, de singular importancia puesto que de ella depende una parte del bienestar cotidiano de todos los habitantes del mundo, especialmente las plantas y los animales, los que proporcionan numerosos elementos que constituyen la base material de la existencia. Esta situación ha devenido preocupación en el hombre contemporáneo, hasta el punto de hacerlo pensar en la necesidad de lograr una actuación ética respecto a su medio ambiente.

\footnotetext{
1 Universidad "José Martí Pérez" Sancti Spíritus, Cuba. Correo electrónico: maite@uniss.edu.cu.

2 Universidad “José Martí Pérez" Sancti Spíritus, Cuba. Correo electrónico: jcesar@uniss.edu.cu.
} 
Diversas son las vías para garantizar la conservación de la biodiversidad y los valores histórico-culturales asociados a ella. Entre estas se destaca por su importancia y viabilidad a largo plazo la conservación in situ, que promueve la protección de ecosistemas y hábitats naturales que merecen especial atención por su alta diversidad y/o fragilidad de las especies. Esta vía comprende el establecimiento y manejo de áreas protegidas y la disposición de un cuerpo legal que garantice el cumplimiento de los objetivos de conservación. Su implementación constituye un objetivo prioritario de la política ambiental nacional y una responsabilidad internacional de la República de Cuba como firmante del Convenio Sobre la Diversidad Biológica.

En la Ley 81 del Medio Ambiente, publicada en la Gaceta Oficial de la República de Cuba (Asamblea Nacional del Poder Popular, 1997), se define la Educación Ambiental como:

...un proceso continuo y permanente, que constituye una dimensión de la educación integral de todos los ciudadanos, orientada a que en la adquisición de conocimientos, desarrollo de hábitos, habilidades, capacidades y actitudes y en la formación de valores, se armonicen las relaciones entre los seres humanos y de ellos con el resto de la sociedad y la naturaleza, para propiciar la orientación de los procesos económicos, sociales y culturales hacia el desarrollo sostenible.

La práctica de la Educación Ambiental, si es efectiva, debe lograr sensibilizar al individuo con los problemas que afectan al medio, dotarlo de conocimientos y desarrollar en él una actitud adecuada en función de resolverlos, de manera que en su interacción armónica con la naturaleza y la sociedad, pueda alcanzar una calidad de vida sostenida y sustentable para las generaciones actuales $\mathrm{y}$ venideras.

El desarrollo sostenible es considerado en la mencionada ley, consultada en la Gaceta Oficial de la República de Cuba (1997) como:

El proceso de elevación sostenida y equitativa de la calidad de vida de las personas, mediante el cual se procura el crecimiento económico y el mejoramiento social, en una combinación armónica con la protección del medio ambiente, de modo que se satisfagan las necesidades de las actuales generaciones, sin poner en riesgo las de futuras generaciones (Ibíd.. p. 49).

El tema relacionado con la Educación Ambiental ha sido tratado con profundidad en varias conferencias realizadas por especialistas, en fórum internacionales, entre los que se citan: La Conferencia sobre Medio Humano, Suecia junio (1972), Las conferencias realizadas en Belgrado (1975), Tbilisi (1977), Moscú (1987, Comisión Mundial sobre Medio Ambiente y desarrollo de la ONU (1987), Convención de Viena para la protección de la capa de ozono (1985), Conferencia sobre Medio Ambiente y desarrollo realizada en Río de Janeiro en (1992), entre otros.

Del análisis realizado se asume que para lograr un nivel de conocimiento sobre la Educación Ambiental con un apreciable nivel de salud, en medio de este mundo globalizado de hoy, se necesita desarrollar en la población, desde su niñez, sentimientos de amor por el cuidado de las plantas, el agua, el aire, desarrollar estados de ánimos relacionados con los problemas ambientales que nos afectan.

Hoy día los problemas medioambientales constituyen una realidad de carácter global, por tanto es importante agrupar la sociedad como fuerza de acción activa en la solución de los mismos, proponiendo acciones que sirvan sobre todo a la educación, pues es ella quien aporta la visión sintetizadora que permite comprender la relación del hombre con su medio.

Para tales logros hay que desarrollar en los individuos nuevas formas de ver el medio a través de una educación conceptual y actitudinal que permita el desarrollo de una actitud ambiental positiva. Juega un papel importante en esto la forma y estilos de pensar, el comportamiento de los individuos, así como su voluntad política.

La población local constituye un componente principal y activo en la conservación y uso sostenible de los recursos naturales. Sin embargo, no siempre desarrollan valores y actitudes positivas hacia el entorno, provocado en gran medida (por una insuficiente adecuada educación), que informe (suficientemente) sobre la importancia de los valores que representan estas áreas y los beneficios que reportan.

Entre las posibles vías para la solución de este problema se encuentra la promoción de una 
Educación Ambiental que propicie a los miembros de la sociedad conocimientos relacionados con el aprovechamiento y el cuidado de los recursos ambientales, facilitando así la utilidad y durabilidad del ecosistema.

\section{Revisión de literatura}

\section{Concepciones históricas y filosóficas que sustentan la investigación}

La Educación Ambiental dentro de la comunidad debe proponer acciones prácticas para lograr que los individuos y pobladores analicen críticamente lo ecológicamente insostenible y socialmente injusto del mundo en que se les propone vivir y se pronuncien como decisores para comenzar a generar cambios en su actuación local que luego trasciendan a escala mundial.

Estas acciones son precisas para evitar la pérdida de la diversidad biológica, cultural y social e impedir la generalización de estilos de vida inadecuados, entendidos como "la contraposición a aspectos cuantitativos y cualitativos que tipifican la calidad de vida del individuo referidos a las condiciones económicas, sociales, políticas, culturales y ecológicas de su existencia... "Carvajal (2000: p. 20) y entre los que pueden citarse el uso indebido de los recursos naturales, el vertimiento inadecuado de desechos sólidos, la pesca ilícita. Estilos estos contrapuestos a la sostenibilidad, eficiencia, durabilidad, suficiencia y equidad cuyo fomento se hace tan necesario en la sociedad.

Según Sánchez y Guiza (1990), la Educación Ambiental "...es un proceso educativo permanentemente orientado a lograr como meta final la participación de los ciudadanos en la prevención y solución de problemas ambientales transformando así, su medio ambiente en función de elevar a planos superiores la calidad de vida de la población. Es la educación que debe orientar hacia un desarrollo sostenido..." Sánchez, (1990.p.4). Actualmente millones de personas sufren las consecuencias de la contaminación ambiental, de la deforestación, del calentamiento global y los peligros que trae consigo la destrucción de la capa de ozono y la desaparición de especies en detrimento de la biodiversidad.

La conservación de una calidad ambiental y la aplicación racional de los avances de la ciencia y la técnica han de complementarse mutuamente con la conformación de un nuevo orden económico más justo y equitativo en el mundo de hoy (UNESCO, 1996).Todo lo anterior demuestra que la capacidad y la consciencia del hombre para cuidar y proteger el planeta, se ha quedado rezagado con respecto a su capacidad y la necesidad de explotarlo y si no se toman medidas a tiempo para impedirlo, el hombre edificará su propia destrucción en un futuro cercano.

En el empeño de lograr que la Educación Ambiental juegue su papel primordial en la protección del ambiente se requiere desarrollar en niños, adolescentes y jóvenes conocimientos, valores, actitudes que los conviertan en ciudadanos con una adecuada cultura ambiental capaz de traducirse en el mejoramiento de la situación contemporánea.

Según Mc Pherson (1997), "La Educación Ambiental en la formación de docentes". (p.14), plantea que conjuntamente, con los objetivos y los principios se tienen en cuenta los fines de la Educación Ambiental, a partir de los cuales y sobre la base de los documentos rectores, se concretan las acciones de Educación Ambiental. Los fines son los siguientes:

- Fomentar una ética ambiental.

- Formar ciudadanos que tengan una comprensión de la relación de la humanidad con todo el ambiente.

- Suministrar información exacta y actualizada acerca del ambiente y sus problemas conexos, para una correcta toma de decisiones.

- Crear incentivo y dar una formación que permita a los ciudadanos adquirir y divulgar conocimientos capaces de ayudar a la sociedad a resolver los problemas ambientales.

- Buscar equilibrio entre las necesidades a corto mediano y largo plazo.

- Hacer que cada ciudadano adquiera un compromiso permanente de protección del medio ambiente.

- Hacer comprender la relación que existe entre las necesidades de la sociedad y su interacción con el ambiente.

- Contribuir a que los ciudadanos estén bien informados de las posibles consecuencias que pueden tener los problemas ambientales.

- Desarrollar el pensamiento crítico.

La forma irracional de la interacción entre el hombre y el medio ambiente, genera los llamados problemas ambientales. No es el desarrollo científico-técnico por sí mismo el causante de los daños 
al medio ambiente, sino, insistimos, su empleo irracional por el principal componente: el hombre. La Educación Ambiental es comunitaria por excelencia, debido a que la comunidad es su campo de acción fundamental, y son los problemas de la comunidad los que constituyen el contenido de sus actividades (Santana y Ortega; 2010).

Por tanto la familia, la escuela, junto con el resto de los factores de la comunidad, son los responsables de enseñar a amar, cuidar, respetar y proteger el medio ambiente. La protección del medio ambiente es un problema estatal, comunitario, familiar y personal. La Educación Ambiental desempeña una función muy importante en la personalidad ambientalmente formada.

\section{Materiales y métodos}

El trabajo fue realizado tomando como referencia 375 habitantes de la comunidad Natividad del municipio La Sierpe, donde se toma como muestra 145 habitantes de la comunidad, los que se dividieron en tres grupos: el centro de la comunidad, el de las zonas aledañas y el apartado, donde cada grupo representa un $15 \%$ para un total de $45 \%$, ya que los 3 grupos intervienen en el uso indiscriminado del medio ambiente, afectan la comunidad y la zona costera del municipio. Para ello se utilizaron los siguientes métodos de investigación:

Análisis - síntesis, inductivo - deductivo; presentes en diferentes momentos del trabajo, desde su concepción hasta la valoración de los posibles resultados, lo cual permite un análisis de las potencialidades que brinda la Educación Ambiental y los objetivos del trabajo en el proyecto institucional del Centro Universitario Municipal (CUM) para su vinculación, la interpretación de datos y de toda la información concerniente a la temática abordada para profundizar en su esencia, partiendo del objetivo propuesto. Histórico - lógico, para sistematizar los antecedentes de la Educación Ambiental y la relación sociedad-naturaleza.

También se utilizaron los métodos del nivel empírico que a continuación se relacionan: Revisión bibliográfica para la fundamentación teórica de la investigación y la elaboración de acciones. El análisis documental, necesario para la revisión de documentos normativos, la Educación Ambiental y el objetivo de trabajo del proyecto institucional del CUM, sustentos básicos de las acciones.

La observación fue útil en el desarrollo del trabajo para determinar actitudes, comportamientos, habilidades de los habitantes acerca de la temática ambiental.

La encuesta con el propósito de determinar las necesidades y motivos que están en la base de la preparación de la Educación Ambiental; así como para determinar el grado de desarrollo de los recursos intelectuales y afectivos que tienen en diferentes momentos al compartir espacios en los que se establece la comunicación entre los miembros del grupo con otras personas, al tratar temas de Educación Ambiental, que sirven como base metodológica de la intervención educativa.

La entrevista individual para constatar que problemáticas concebía este grupo como necesidad urgente a resolver y con qué potencialidades contaban los habitantes de la comunidad Natividad del Municipio La Sierpe.

La entrevista grupal a la muestra para conocer el estado acerca del conocimiento en la temática ambiental de los habitantes de la comunidad Natividad del Municipio La Sierpe.

La entrevista en profundidad, para valorar el impacto de las acciones culturales y medioambientales aplicadas en la comunidad.

Por otra parte, fue necesario dentro del nivel matemático - estadístico el cálculo porcentual como procedimiento matemático.

Las categorías de análisis que emergieron en el proceso investigativo fueron las siguientes: Contenido de la cultura medioambiental, el trabajo en equipo, la comunicación interpersonal, que se analiza en primer lugar por la posición de cada individuo hacia el entorno, la toma de decisiones, el sentimiento de amor a la naturaleza y el respeto a la diversidad.

\section{Resultados y discusión}

La relación hombre-naturaleza deviene una decisión incuestionable, al considerar que ha sido esta Ciencia la que ha expresado las características de las comunidades humanas desde la óptica de sus relaciones con el entorno natural. Permite, además, especificar y generalizar aspectos de la complejidad ambiental.

¿Cómo han reflejado los hombres --a lo largo de la humanidad - sus modos de vida, sus modelos 
de relación con la naturaleza, sus preocupaciones por los cambios ocurridos en su medio ambiente?

Aunque en las circunstancias actuales se han agudizado los problemas en la sociedad, y el medio ambiente no es objetivo plantear que son problemas de la contemporaneidad. Sería más correcto decir que tales problemáticas poseen "raíces, quizás, desde la aparición del Homo sapiens; desde entonces ya comienzan a visualizarse los gérmenes de la relación contradictoria sociedad naturaleza (...)".

En tal sentido, Francesco di Castri considera que: “...ya entre los romanos encontramos elementos de principios ecológicos en las obras del filósofo Lucrecio, del poeta Virgilio o del agrónomo Columela, pero también la hallamos en todas las demás civilizaciones de la antigüedad."

¿Cuáles fueron las primeras apropiaciones cognoscitivas del hombre hechas de la naturaleza?

"En cuanto modificador de la composición de las especies dominantes, data de hace unos 7500 años. Hace 3500 se producían ya importantes fenómenos de deforestación."

Los problemas relativos a la naturaleza han sido un asunto de interés para muchos hombres, desde la antigüedad hasta la actualidad. Para Tales de Mileto (624-547 a.n.e.) el agua era el material del cual todo se generaba; para Anaxímedes (585-525 a.n.e.) era el aire; para Heráclito (530-470 a.n.e.) el fuego y para Aneximandro (610-548 a.n.e.) el apeirón, principio resultante de la unidad de los elementos esenciales en la naturaleza. Empeódocles (504-443 a.n.e.) consideró que los cuatro elementos constituyentes del mundo eran el fuego, la tierra, el agua y el aire, y estableció una relación armoniosa entre ellos. Según él, la falta de armonía y la existencia de un desequilibrio entre estos elementos, causaba enfermedades y la muerte.

Otros pensadores como Hipócrates (S. V a.n.e.) expusieron sus tesis sobre el determinismo geográfico o medioambiental, a partir de lo cual consideraron que era necesario establecer una comprensión de la relación sociedad-naturaleza, dado los vínculos existentes entre ellos.

La labor de José Agustín Caballero como iniciador de la reforma filosófica en Cuba, donde según sus palabras su filosofía es "portadora de un método que lejos de adivinar los secretos de la naturaleza, anticipándose a ella, la interroga por medio de la experiencia, y la estudia con observaciones continuas y bien meditadas, con lo cual, cada nuevo descubrimiento tiene una aplicación que redunda en provecho de la sociedad".

Fue ese entendimiento del papel de la naturaleza en la cultura y en la sociedad -visión proveniente del pensamiento fundacional cubano, a partir de Félix Varela y de José Martí, y continuado en las ideas por Fidel.

Martí (1883) dictó: -Divorciar el hombre de la tierra, es un atentado monstruoso. Y eso es meramente escolástico: ese divorcio. -A las aves, alas; a los peces, aletas; a los hombres que viven en la Naturaleza, el conocimiento de la Naturaleza: esas son sus alas. Y el medio único de ponérselas es hacer de modo que el elemento científico sea como el hueso del sistema de educación pública.

Como se ha demostrado a lo largo de la historia humana, los hombres han mantenido una visión de entendimiento y preocupación para con la naturaleza y la sociedad; esta última posee un solo espacio para su construcción: el espacio natural. Mientras tanto, las influencias de los seres humanos han mantenido el curso del consumo de los recursos naturales, unos ya agotados, otros por agotarse, actitud latente en nuestros días y con un ascenso vertical a pesar de los diversos compromisos adoptados por las naciones participantes en la Cumbre de Río, en el año 1992.

¿Qué tipo de acciones puede mejorar la calidad de la relación sociedad-naturaleza en el sentido de ofrecerle a esta última la capacidad de reproducirse en su totalidad?

Sería bueno considerar y asumir que la complejidad de la Educación Ambiental precisa de intervenciones educativas que no pretendan abarcarlo todo y sí hacerle frente con rigor científico a una sola arista, aunque existe el consenso de que es muy difícil influir en una, sin que ésta repercuta en otras, debido al carácter dialéctico de la personalidad y las múltiples relaciones entre las distintas categorías psicológicas.

En intercambio con los habitantes vinculado al cuidado y protección del medio ambiente de manera general, se pudo constatar que una de las razones que más se destacan entre otras, es la falta de conocimiento adecuado para proteger el medio ambiente, dificultad que viene dada desde su formación, por la exigencia de los tiempos que 
vive el país, teniendo en cuenta el deterioro de las condiciones ambientales, tanto en el ámbito global como local no se puede dejar a la espontaneidad, es por tanto imprescindible incorporarse a los trabajos con la comunidad, acciones bien estructuradas que respondan a las necesidades reales respecto a cuáles son los conocimientos de los que deben pertrecharse los decisores y habitantes de la misma, a partir de que en este momento estos no tienen la formación necesaria.

Durante el diagnóstico se comprobó que los habitantes y decisores de la comunidad carecían hasta este momento del conocimiento que necesitaban en materia de Educación Ambiental, cambio de actitud en las personas para ser consecuentes en la conservación del medio ambiente.

En resumen, las principales deficiencias que se manifestaron en el diagnóstico fueron:

1. No existe la claridad necesaria del concepto de medio ambiente en parte significativa en la comunidad.

2. Los directivos no tienen la preparación adecuada en materia de conservación Medio Ambiental.

3. No se realizan acciones para conservar el Medio Ambiente, en el territorio, a pesar de contar con la Estrategia Municipal de Medio Ambiente.

4. Las actividades en la comunidad carecen de la dimensión ambiental y por tanto no las proyectan para que tributen a su desarrollo.

5. Falta de información de los problemas ambientales de nivel mundial, nacional, provincial, municipal y de la comunidad.

6. Falta de divulgación desde las instituciones culturales y en los territorios de la problemática ambiental.

Durante entrevistas realizadas a los habitantes de la comunidad y sus decisores, se evidenció, que históricamente no existía suficiente conocimiento de la Educación Ambiental. Ellos plantearon, que es necesario el desarrollo de actividades que traigan como resultado la interiorización de una cultura ambiental, en la que todas las actividades de Educación Ambiental que se desarrollen, confluyan en acciones conscientes, para que la población y los decisores actúen en consecuencia con la preservación del medio ambiente.
Las personas consultadas hicieron además un grupo de sugerencias, entre las cuales se encuentran:

1. Realización de talleres, cursos, conferencias $y$ otras acciones con objetivo de elevar el conocimiento sobre la Educación Ambiental.

2. Incrementar la divulgación ambiental a través de los medios de comunicación.

¿Cómo diseñar acciones culturales y medio-ambientales para los habitantes dela comunidad Natividad del municipio La Sierpe?

Para diseñar estas acciones, se tuvo en cuenta las carencias sobre la temática ambiental de los decisores y habitantes de la comunidad, las que se detectaron a través de la aplicación del diagnóstico.

Una vez que ya conocidas e identificadas dónde están las principales dificultades en la comunidad, se debe proceder a la planificación de los requisitos a tener en cuenta para la organización de una propuesta de acciones, específicamente dirigido al cuidado y protección del medio ambiente y otros temas colaterales.

De este modo, se van sucediendo las acciones en que el hombre y el medio ambiente son los dos elementos fundamentales de la comunidad, pero se manifiesta el hecho, de que el ser humano sigue ejerciendo su acción depredadora en las más insospechadas formas. Por lo que deben de acometerse acciones de manera que todas las personas aporten lo que les corresponde en este concierto organizado, en el que sólo se escuche la voz de la razón imponiéndose por salvar lo que por derecho nos corresponde defender.

Seguir modelos que conduzcan al desarrollo sostenible, teniendo presente relaciones como la relación sociedad-naturaleza, al efectuarse el tratamiento de esta idea es importante que se comprenda las intensas, complejas, continuas y bilaterales acciones entre el hombre la naturaleza y la sociedad; que esta interacción es dinámica y se desarrolla en el tiempo y el espacio evidenciando su complejidad, por lo que debe propiciar la visión integral del individuo acerca del medio ambiente y la educación.

En lo referido a la protección del hombre y el mejoramiento de la calidad de vida, es evidente que 
la relación que se establece entre el medio ambiente y el hombre como ser biológico y social repercute en todas las esferas de su vida cotidiana en la interacción con el medio ambiente.

La responsabilidad del hombre ante el medio ambiente está encaminada al desarrollo de actuaciones responsables en su vida personal y profesional, por tanto, indisolublemente relacionada con la formación de valores. "Se requiere que siempre que se analice un problema o situación ambiental se determine las causas, consecuencias y alternativas de solución".

Estas acciones, la conformamos atendiendo a la forma en que se pueda llevar a vías de hecho los habitantes, en el cual los mismos, sean capaces de apropiarse de conocimientos, a tener en cuenta, en el momento de accionar dentro de la comunidad y se deben someter al proceso de Evaluación de Impacto Ambiental, según lo establece la Ley 81/97 y la Resolución 77/99 (CITMA, 1999).Es requisito indispensable, para los directivos en general guiar a nuestras generaciones actuales y futuras por el camino de pertrecharnos de una educación y cultura ambiental, sobre la base de los resultados de un proceso de concientización arraigado en las más profundas raíces de cada uno de los miembros de nuestra sociedad.

Es por tal motivo, que nuestra investigación consiste en la presentación de acciones, en la que se integran los conocimientos generales necesarios, comprendidos los principales conceptos como: la política, Educación Ambiental, desarrollo sostenible, entre otros, y los elementos de cada herramienta legal, a tener presente en todos los momentos, así como también durante la ejecución, etapa en la que siempre debe de tenerse un control exacto, de los requerimientos impuestos y que deben ser cumplidos rigurosamente.

Si la población posee los conocimientos esenciales, adquiridos mediante algunas de las formas de superación profesional, pero no posee los conocimientos necesarios sobre los métodos y procedimientos para la solución de un problema, se requiere que su superación se efectúe en consonancia con sus propias dificultades.

De esta forma, se aprecia lo importante que resulta ejecutar acciones en torno al desarrollo de habilidades, hábitos, capacidades que propicien el conocimiento y permitan elevar una conciencia ambiental.

\section{Bibliografía}

Asamblea Nacional del Poder Popular (1997). Ley 81 del Medio ambiente. Publicado en La Gaceta oficial de la República de Cuba, La Habana.

Caballero, José Agustín (1944). Philosophia Electiva. Editorial Universidad de la Habana.

Carvajal Rodríguez, Cirelda (200o). Educación para la salud en la escuela. La Habana: Ed. Pueblo y Educación.

Castro Ruz, Fidel (1992). Discurso pronunciado en la Conferencia de las Naciones Unidas sobre Medio Ambiente y Desarrollo, Río de Janeiro. 1 Periódico Granma, Junio 5. 1992.

Di Castri, F./. (1981). La ecología moderna: génesis de una ciencia del hombre y la naturaleza.

Luz y Caballero, José de la (1946). La Polémica Filosófica. Edit. de la Universidad de la Habana.

Martí, José (1883). Educación Científica, en la América, Nueva York, septiembre de 1883.O.C., t.8, p.278.

Ministerio de Ciencia, Tecnología y Medio Ambiente (1999). Reglamento del proceso de evaluación de impacto ambiental. Resolución No. 77/99.Habana, Cuba.

Ph Pherson Sayú Margarita. (1997). Estrategia y metodología de la Educación Ambiental en la formación de profesores. La Habana, Edición IPLAC.

Sánchez, Vicente y Guiza. (1990). Glosario de términos sobre Medio Ambiente. Santiago de Chile: UNESCO, OREAL.

Santana Esponda, Yamila y Ortega Vera Ramiro Renol (2010). Orientación sobre Educación Ambiental para los profesores en formación de la enseñanza media superior. IPI. Ramón Ribalta Martínez". agua La Grande. Villa Clara. Cuba.

Tbilisi (1977). Informe final. París.

UNESCO - PNUMA. (1996). Tendencias, necesidades y prioridades en la Educación Ambiental desde la conferencia de Tbilisi. Publicado por la Oficina Regional de Educación para América Latina y el Caribe (OREALCE). Santiago de Chile. 